Original article

\title{
Elastic modulus of the femoral trochanteric region measured by scanning acoustic microscopy in elderly women
}

Hiroyuki Matsuki · Junichi Shibano · Michiaki Kobayashi · Yukio Nakatsuchi ·

Tetsuji Moriizumi · Hiroyuki Kato

H. Matsuki (Corresponding author) $\cdot$ H. Kato

Department of Orthopaedic Surgery, Shinshu University School of Medicine, 3-1-1

Asahi, Matsumoto, Nagano 390-8621, Japan

J. Shibano $\cdot$ M. Kobayashi

Department of Mechanical Engineering, Kitami Institute of Technology, 165 Koen-cho, Kitami, Hokkaido 090-8507, Japan

Y. Nakatsuchi

Department of Orthopaedic Surgery, Marunouchi Hospital, 1-7-45 Nagisa, Matsumoto, Nagano 390-8601, Japan

T. Moriizumi

Department of Anatomy, Shinshu University School of Medicine, 3-1-1 Asahi, Matsumoto, Nagano 390-8621, Japan

\section{Corresponding author:}

Hiroyuki Matsuki

Shinshu University School of Medicine

3-1-1 Asahi, Matsumoto, Nagano 390-8621, Japan

Tel.: +81-263-37-2659

Fax: +81-263-35-8844

E-mail: matsuki_h@marunouchi.or.jp 


\section{Abstract}

Purpose We have devised a method that can obtain continuous detailed distributions of the elastic modulus along the measurement line in a non-decalcified specimen of human bone tissue. The aim of this study was to determine whether local variations exist in the distribution of mechanical properties within the trochanteric region of the femur of elderly females using a newly developed form of scanning acoustic microscopy (SAM) technology.

Methods Human proximal femurs were harvested from 7 female cadavers aged between 67 and 88 years at death. Using the collected data with SAM, elastic modulus of cortical and trabecular bone tissue of the lateral and medial trochanter was calculated and statistically analyzed.

Results The longitudinal and transverse elastic moduli in cortical bone tissue of the lateral trochanter were found to be significantly lower than those of the medial trochanter in all specimens from individuals over age $70(\mathrm{p}<0.05)$. Compared to that of the distal region, the longitudinal and transverse elastic moduli of trabecular bone tissue of the proximal region of the lateral trochanter were significantly lower in all specimens from individuals over age $80(\mathrm{p}<0.05)$.

Conclusion Our new method allows obtaining detailed distributions of the elastic modulus of bone tissue.

Keywords Scanning acoustic microscopy $\cdot$ Elastic modulus $\cdot$ Bone tissue $\cdot$ Trochanter 


\section{Introduction}

It is generally accepted that bone strength is closely related to not only bone mass but also bone quality, the latter of which is widely considered to affect fracture risk. Assessment of bone quality is widely performed using mechanical testing techniques, such as uniaxial tensile, compressive, and 3-point or 4-point bending testing, that measure the mechanical properties of a bone specimen. However, traditional modalities of mechanical testing may not necessarily be adequate when attempting to evaluate the mechanical properties of the bone tissue. The nanoindentation technique [1-3], finite element modeling [4-7], and scanning acoustic microscopy (SAM) technique [2, 8-11] have been commonly used to characterize the tissue-level elastic properties of cortical and trabecular bone. Among these measurement modalities, SAM technique offer several advantages over direct biomechanical testing, in particular that of allowing for measurement in a non-invasive and non-destructive manner at a high resolution, generally in the range of 1 to $10 \mu \mathrm{m}$.

Among the factors identified as risk factors in the health of elderly populations, hip fracture has been identified as the most serious, particularly in individuals with osteoporosis. Most frequently occurring during low-energy injures, such as falls, this type of fracture has long been recognized as the most serious consequence of osteoporosis. Hip fracture has not only been found to result in impairment in activities of daily living, morbidity, and shortened life expectancy, with a reported mortality rate in the year following hip fracture of approximately 30\% [12], but also to impose a huge financial burden on healthcare systems [13]. A hip fracture is generally classified as either cervical or trochanteric in accordance with its anatomic location. Although it is known that the rates of trochanteric fracture increase dramatically with age in the elderly female population, $[14,15]$, the reason for this phenomenon remains unclear. Based on consideration of this association, this study developed and tested the hypothesis that subtle differences in the local distribution of mechanical properties within the trochanteric region may be related to the increase of trochanteric fracture with age.

We have devised a method that can obtain continuous detailed distributions of the elastic modulus along the measurement line in a non-decalcified specimen of human bone tissue. The elastic modulus calculated with our method indicates the average value of the elastic modulus in any direction without considering the anisotropic properties. In general, the mechanical properties of the bone are anisotropy. However, we consider that it is worth applying an isotropic approximation to obtain clinically useful information because the measurements are made under the same condition and within 
the same sample. The aim of this study was to test whether elderly females present local variations in the distribution of elastic modulus within the trochanteric region of the femur using a new adaptation of the SAM technology.

\section{Materials and methods}

Subjects

The new SAM method was validated using human proximal femurs obtained from 7 female cadavers without history of bone-related disease except osteoporosis, ranging in age from 67 to 88 years old.

Preparation of specimens

The proximal femur was coronally sectioned into halves across the center of neck using a precision cutting machine (Acutome 5, Struers, Copenhagen, Denmark). The trochanteric region was obtained from each half section. The trimmed specimen was then fixed with $4 \%$ formalin solution for 4 days before being embedded in epoxy resin consolidated at a cure temperature of $25^{\circ} \mathrm{C}$ (Scandiplex, Scandia, Hagen, Germany). The surface of the coronal section was polished with silicon carbide abrasive papers down to 4000 grit and diamond abrasive powder down to $1 \mu \mathrm{m}$ particle size (Struers, Copenhagen, Denmark) in using automatic grinding and polishing machine (CP30, Logitech, Glasgow, UK). The samples were stored in gauze soaked in distilled water at $4^{\circ} \mathrm{C}$ and maintained wet at all times during measurement in order to preserve moisture property of the sample.

Calculation of elastic modulus

SAM was performed using an H-SAM machine (Hitachikenki, Tokyo, Japan) operated at a frequency of $200 \mathrm{MHz}$ at room temperature, with distilled water used as the coupling medium between the lens and the specimen. An ultrasound wave generated from the transducer was converted by the lens and focused on the sample through a coupling liquid, and it traveled along the sub-surface of material with the penetration depth $\lambda_{R}$ that is the wave length of the leaky surface acoustic wave (LSAW) and determined by the LSAW velocity and the frequency. The ultrasound wave reflected back to the lens was transformed by the piezoelectric transducer into a voltage proportional to the ultrasound wave that was stored in the electronic hardware. A 
recording of the voltage can be converted into a 256 level grey-scale image referred to as a $\mathrm{C}$-mode image that illustrates the acoustic impedance $Z$, defined as the product of the local density $\rho$ at the point on the material and the longitudinal acoustic velocity $v$ (i.e., $Z=\rho \times v$ ). Measurement of the velocity of the LSAW (Rayleigh waves) and other mechanical properties is commonly performed using the interference method, also referred to as the $V(z)$ method, which consists of recording the transducer output voltage $V$ with variations in the defocusing distance $z$. Using this method, constructive and destructive interference between surface modes are denoted by peaks and dips in the resulting $V(z)$ curve and the velocity of Rayleigh waves $V_{R}$ is defined as follows [16] :

$$
V_{R}=\frac{V_{W}}{\left[1-\left(\frac{V_{W}}{2 F \Delta Z}\right)^{2}\right]^{\frac{1}{2}}}
$$

where $\Delta Z$ is the period between 2 successive peaks in the $V(z)$ curve, $F$ is the frequency of the ultrasound wave, and $V_{W}$ is the velocity of ultrasonic waves in water.

The H-SAM machine to measure the bone mechanical properties in the current study has an advantage of the high image resolution based on the interference of the longitudinal wave directly reflected from the specimen surface with LSAW propagated along the specimen surface using the time gate method for the wave separation [17]. The lateral and the depth resolutions of $V(z)$ curve are almost $0.4 \lambda_{R}$ and the penetration depth from specimen surface is $\lambda_{R}$, respectively. Due to the bone mechanical properties measured in this study, the LSAW velocity was almost $3500 \mathrm{~m} / \mathrm{s}$, and thus the $\lambda_{R}$ was 18 $\mu \mathrm{m}$, while both the lateral and $V(z)$ depth resolution was almost $7 \mu \mathrm{m}$ under a frequency of $200 \mathrm{MHz}$.

We can reduce the time required for calculating the elastic modulus by using the new method $[18,19]$. The value of the reflection coefficient of bone, $R_{b}$ is defined as follows:

$$
R_{b}=\frac{Z_{L b}-Z_{W}}{Z_{L b}+Z_{W}}
$$

where $Z_{L b}$ is the acoustic impedance of bone and $Z_{w}$ is the acoustic impedance of water in the longitudinal wave. As it is difficult to measure $R_{b}$ accurately using H-SAM machine, the reflection coefficient of aluminum, for which the acoustic impedance for 
the longitudinal wave is already known, is measured under the same conditions. The reflection coefficient of aluminum $R_{a}$ is defined as follows:

$$
R_{a}=\frac{Z_{L a}-Z_{W}}{Z_{L a}+Z_{W}}
$$

where $Z_{L a}$ is the acoustic impedance of aluminum in the longitudinal wave. Using the results of (2) and (3), the reflection intensity ratio $X\left(=R_{b} / R_{a}\right)$ can be calculated using the following formula:

$$
X=\frac{R_{b}}{R_{a}}=\frac{\left(Z_{L b}-Z_{w}\right)\left(Z_{L a}+Z_{w}\right)}{\left(Z_{L a}-Z_{w}\right)\left(Z_{L b}+Z_{w}\right)}
$$

Using the results of (2), (3), and (4), $Z_{L b}$ can be calculated using the following formula:

$$
Z_{L b}=\frac{\left(Z_{L a}+Z_{w}\right)+\left(Z_{L a}-Z_{w}\right) X}{\left(Z_{L a}+Z_{w}\right)-\left(Z_{L a}-Z_{w}\right) X} Z_{w}
$$

Although determining the value of the acoustic impedance of bone in the transverse wave $Z_{T b}$ is necessary to calculate the bone density $\rho_{b}$ and Poisson's ratio $v$, it is impossible to measure $Z_{T b}$ directly because the transverse wave can't propagate through the water coupler. Therefore, an approximate value is obtained by measuring the reflection intensity ratio $X$ of the surface of bone tissue and substituting the value $X$ into the following equation :

$$
Z_{T b}=0.157 e^{4.047 X}
$$

which was derived from the master curve as shown in Fig. 1. The relationship between acoustic impedance in transverse wave $Z_{T}$ and reflection intensity ratio in longitudinal 
wave $X$ was plotted based on the known values of several metals and non-metals normalized by the intensity of a pure Aluminum. From this relationship, the approximate curve and its equation were obtained.

Here, $Z_{L b}$ and $Z_{T b}$ defined by (5) and (6), respectively, have functionally same tendency about the reflection intensity $X$ according to the following relation:

$$
\frac{V_{T b}}{V_{L b}}=\frac{Z_{T b}}{Z_{L b}}=\sqrt{\frac{1-2 v}{2(1-v)}}
$$

To examine the adequateness of (6), we consider the extreme case of $X=0$, the case of the water, in which $Z_{L b}=Z w$ deduced from (5) is approximately $1.5 \mathrm{MPa} \cdot \mathrm{s} / \mathrm{m}$ and $Z_{T b}$ is $0.157 \mathrm{MPa} \cdot \mathrm{s} / \mathrm{m}$ from (6). Substituting the values of $Z_{L b}$ and $Z_{T b}$ into (7), the value of Poisson's ratio $v$ is obtained to be 0.494 which adequately satisfies to be incompressible. Moreover, it is obtained from (5) - (7) that Poisson's ratio is approaching -1.0 under the reflection intensity $X$ to be approaching infinity. Therefore, it is concluded from (5) - (7) that the range of $X: 0 \leq X \leq \infty$ corresponds to the physical range of Poisson's ratio: $0.5 \geq v \geq-1.0$.

As the velocity of Rayleigh surface wave $V_{R b}$ propagating the surface of bone tissue is related to the velocity of bone for transverse wave $V_{T b}$ and longitudinal wave $V_{L b}$ [20], it is calculated as follows:

$$
\left(\frac{V_{R b}}{V_{T b}}\right)^{6}-8\left(\frac{V_{R b}}{V_{T b}}\right)^{4}+8\left(\frac{V_{R b}}{V_{T b}}\right)^{2}\left\{3-2\left(\frac{V_{T b}}{V_{L b}}\right)^{2}\right\}-16\left\{1-\left(\frac{V_{T b}}{V_{L b}}\right)^{2}\right\}=0
$$

with $V_{L b}$ and $V_{T b}$ determined using the value of bone density $\rho_{b}$ and equations (9) and (10), respectively, as follows:

$$
\begin{gathered}
V_{L b}=\frac{Z_{L b}}{\rho_{b}} \\
V_{T b}=\frac{Z_{T b}}{\rho_{b}}
\end{gathered}
$$


$\rho_{b}$ is calculated using (11), a third degree equation of $\rho_{b}^{2}$ obtained by substituting (9) and (10) into (8) as follows:

$$
\left(\frac{V_{R b}}{Z_{T b}}\right)^{6} \rho_{b}{ }^{6}-8\left(\frac{V_{R b}}{Z_{T b}}\right)^{4} \rho_{b}{ }^{4}+8\left(\frac{V_{R b}}{Z_{T b}}\right)^{2}\left\{3-2\left(\frac{Z_{T b}}{Z_{L b}}\right)^{2}\right\} \rho_{b}{ }^{2}-16\left\{1-\left(\frac{Z_{T b}}{Z_{L b}}\right)^{2}\right\}=0
$$

The proposed method to measure the density at the surface sensitively depends on the physical surface state, i.e. the surface roughness and so forth. For example of the bulk Aluminum alloy density $2.69 \mathrm{~g} / \mathrm{cm}^{3}$ (weight: $8.6 \mathrm{~g}$, volume: $3.2 \mathrm{~cm}^{3}$ ) the average of the densities at 10 points of the surface measured by the proposed method was $2.75 \mathrm{~g} / \mathrm{cm}^{3}\left(2.78,2.71,2.78,2.71,2.90,2.81,2.79,2.56,2.54\right.$ and $2.91 \mathrm{~g} / \mathrm{cm}^{3}$ in detail) and therefore, the dispersion between the bulk and the surface densities was almost under $10 \%$.

After determining the Poisson's ratio $v$ using equation (7), the shear modulus (elastic modulus in the transverse direction) $G_{b}$ and Young's modulus (elastic modulus in the longitudinal direction) $E_{b}$ can be calculated as follows:

$$
G_{b}=\frac{Z_{T b}^{2}}{\rho_{b}}
$$

$$
E_{b}=2(1+v) G_{b}
$$

SAM has C-mode function to display the surface image of a specimen. C-mode image consists of $400 \times 400$ pixels, and the luminance value of it with 256 gray scale levels is proportional to the magnitude of the ultrasonic reflection echo. And, the ultrasonic reflection coefficient relevant to the mechanical characteristics of specimen shown in equation (2) is also proportional to the ultrasonic reflection magnitude. Then, the authors of this study devised a method to estimate conversely the reflection coefficient from the luminance value of the C-mode image [21]. The relation between the ultrasonic reflection magnitude of the bone tissue and the luminance value of $\mathrm{C}$-mode image of it can be determined as follows. Since a canine bone has the almost same mechanical properties as a human bone, it was used for a measurement reference 
of bone. C-mode image of the diaphysial cortical bone of the femur of the canine (male, 28 eight months old) was shown in Fig. 2. The magnification of SAM was adjusted so that the size of one pixel of C-mode image became to $10 \mu \mathrm{m}$ which was slightly larger than the resolution of SAM. The acoustic lens of SAM was positioned on the point that a cortical bone tissue exists in the specimen. As the ultrasonic reflection magnitude indicates the value of about $4.0 \mathrm{~V}$, the magnitude of incident ultrasonic wave was controlled with the output power of the transmitter of SAM. The luminance value of the point was obtained from the C-mode image data, and the ultrasonic reflection magnitude in the same point was measured simultaneously. The output power of the transmitter was decreased gradually and the same measurement was repeated in the same position. The relation between the luminance value of $\mathrm{C}$-mode image and the ultrasonic reflection magnitude of the specimen was obtained as shown in Fig. 3. From the dispersion in the data of the graph, the ultrasonic reflection magnitude calculated from the approximate line contained about $\pm 0.0676 \mathrm{~V}$ as the standard error of estimates. Then, the empirical formula which indicates the relation between the luminance value $L$ and the reflection coefficient of the bone $R_{\mathrm{b}}$ can be obtained as follows:

$$
R_{b}=0.013 L+d
$$

Constant $d$ is determined by measuring $R_{b}$ and $L$ on the same point on the measurement line in calculating the velocity of Rayleigh waves $V_{R}$ in the $\mathrm{X}-\mathrm{Z}$ plane and substituting their values into equation (14). The difference between the ultrasonic reflection magnitude measured directly and the value obtained by this method was $2 \%$ or less at the maximum. The use of this method not only reduces the amount of time required to measure $R_{b}$ in comparison with conventional methods but also enables measurement of 400 points of $R_{b}$ on each measurement line, allowing for efficient and continuous measurement of the distributions of elastic modulus along the measurement line.

The measured data were obtained by $\mathrm{C}$-mode and $\mathrm{X}-\mathrm{Z}$ mode, namely the luminance value was obtained by $\mathrm{C}$-mode and the LSAW velocity was obtained by $\mathrm{X}-\mathrm{Z}$ mode, respectively. Therefore, the measured areas of C-mode and X-Z mode observed by the one scan are $4 \mathrm{~mm}$ square and $4 \mathrm{~mm}$ length $\mathrm{x} 10 \mu \mathrm{m}$ width rectangular, respectively. According to the $4 \mathrm{~mm}$ square measured area of each SAM scan measurement represented by $400 \times 400$ pixels in the C-mode image, one pixel corresponds to $10 \mu \mathrm{m}$ square of the specimen area. Therefore, the spatial resolution of the measurements is 10 $\mu \mathrm{m}$ and it is the interval of each SAM scan measurement. To calculate one elastic 
modulus, the values of 2 pixels of C-mode image and X-Z-scan data were needed. Therefore, the measured area to obtain one elastic modulus is always $20 \mu \mathrm{m} \times 10 \mu \mathrm{m}$ rectangular specimen area.

Measurement of elastic modulus

Measurement of the elastic modulus of both the lateral and medial trochanter was performed because the fracture line was located at these regions in the trochanteric fracture. Table 1 shows the measurement area of the trochanteric regions. Cortical bone and trabecular bone were scanned perpendicular to the axis of femoral shaft from the tip of the greater trochanter to the level of the upper rim of the lesser trochanter in the lateral trochanter with 16 lines scanned each of $4 \mathrm{~mm}$ scan length at regular intervals and from the upper rim to the lower rim of the lesser trochanter with 10 lines in the medial trochanter as illustrated in Fig 4.

Statistical analysis

Data were analyzed using SPSS Statistics software (SPSS, Chicago, USA) and measured values statistically expressed as means and standard deviations. The Mann-Whitney U test was used to determine the significance of differences between 2 groups and the level of statistical significance assigned to $p$ values was $<0.05$.

\section{Results}

Elastic modulus of the trochanteric region

The elastic modulus of the cortical and trabecular bone tissue in the lateral and medial trochanter was measured, as the fracture line was located at these regions in the trochanteric fracture, and elastic modulus of the lateral and medial trochanteric region were compared to detect the reason for the increase of the rates of trochanteric fracture with age in the elderly female population. In the lateral trochanter, average number of scanned data to obtain one elastic modulus was 9.0 in average ranging from 2 to 27 in cortical bone tissue and 8.8 from 2 to 24 in trabecular bone tissue, respectively. In the medial trochanter, average number of scanned data to obtain one elastic modulus was 27.8 from 8 to 58 in cortical bone tissue and 17.4 from 3 to 47 in trabecular bone tissue, respectively. Table 2 shows the results of the measurement of the elastic modulus and bone density of the trochanteric region. In cortical bone tissue, the mean elastic modulus 
in the longitudinal direction ranged from 14.26 to $22.53 \mathrm{GPa}$ in the lateral trochanter and from 23.4 to $35.28 \mathrm{GPa}$ in the medial trochanter, while that in the transverse direction ranged from 5.07 to $8.23 \mathrm{GPa}$ in the lateral trochanter and from 8.52 to 13.38 $\mathrm{GPa}$ in the medial trochanter. In trabecular bone tissue, the mean elastic modulus in the longitudinal direction ranged from 14.84 to $22.55 \mathrm{GPa}$ in the lateral trochanter and from 18.27 to $28.17 \mathrm{GPa}$ in the medial trochanter, while that in the transverse direction ranged from 5.24 to $8.18 \mathrm{GPa}$ in the lateral trochanter and from 6.67 to $10.5 \mathrm{GPa}$ in the medial trochanter. As described above, the longitudinal and transverse elastic moduli of the cortical bone tissue of the lateral trochanter of all specimens from individuals over age 70 were found to be significantly lower than that of the medial trochanter as shown in Fig. $5(\mathrm{p}<0.05)$.

The elastic modulus of proximal regions (line 1-8) and distal regions (line 9-16) of the lateral trochanter was compared with respect to the elastic modulus of trabecular bone tissue to investigate specificities of the local distribution of mechanical properties at the lateral trochanteric region with a peculiar configuration. Table 3 shows the results of comparison of the mean elastic modulus of trabecular bone tissue in the proximal and distal regions of the lateral trochanter. As can be observed, the longitudinal elastic modulus of the trabecular bone tissue of the proximal region was relatively low in all individuals aged between 70 and 89 compared to that of the distal region, while the transverse elastic modulus was relatively low in 2 individuals aged between 70 and 79 and in 3 individuals aged between 80 and 89 compared to that of the distal region. Statistical analysis revealed a significant difference in the longitudinal and the transverse elastic moduli between the proximal and distal regions in all individuals aged between 80 and 89 as shown in Fig. $6(\mathrm{p}<0.05)$.

\section{Discussion}

Measuring the mechanical properties of trabecular bone tissue is more difficult than measuring those of cortical bone tissue because of the extremely small dimensions of the individual trabeculae in trabecular bone. Among the various methods that have been used to measure tissue-level elastic properties of trabecular bone, the nanoindentation technique [1-3], finite element modeling [4-7], and ultrasonic techniques $[2,8-11]$ have been the most widely used over the past 20 years. The nanoindentation technique, which is used for simultaneously measuring the force and displacement of a diamond indenter impressed into a material specimen, was recently improved to address the nonlinear nature of the unloading load-displacement curve for nonflat indenters. Use of the nanoindentation technique provides the tremendous 
advantage of allowing for probing of bone tissue properties at the microscopic level. In one study using the nanoindentation technique, the average elastic modulus was found to be $13.5 \pm 2.0 \mathrm{GPa}$ for the human vertebral trabecular bone tissue, $22.5 \pm 1.3 \mathrm{GPa}$ for osteons, and $25.8 \pm 0.7 \mathrm{GPa}$ for interstitial bone tissue of tibial cortical bone [1] Although calculation of the Young's modulus using the nanoindentation technique has been reported to be relatively insensitive to the chosen value for Poisson's ratio [2], it has also been reported that the elastic modulus value obtained from the use of the nanoindentation technique is highly affected by the indentation rate and extent of time-dependent plasticity [22].

Another approach to mechanical testing at the tissue level of bone is use of high-resolution finite element models constructed using the data derived from micro-computed tomography or micro-magnetic resonance imaging. Specimen-specific experimental data at the whole specimen level were employed as the material modulus to calibrate effective elastic properties of the tissue. It has been shown that models constructed using this procedure can provide accurate predictions of the mechanical properties of trabecular bone at a continuum level [23]. Although previous finite element studies of human vertebral trabecular bone have reported relatively low effective tissue moduli values of 5.7 to $6.6 \mathrm{GPa}[4,5]$, recent studies of trabecular bone tissue have reported much higher values of 18.0 to $18.7 \mathrm{GPa}[6,7]$. In most of studies using the finite element technique, compression testing has been used with platens to provide the calibration data. Therefore, because of the associated end artifacts [24, 25], most studies using high-resolution finite element models have reported low values of the calibrated effective tissue moduli compared to experimental studies using the nanoindentation or ultrasonic techniques.

Since the mechanically SAM was first introduced by Lemons and Quate in 1975 [26], it has become a valuable tool in bone research. SAM technique offers several advantages over direct biomechanical testing, in particular that of allowing for measurement in a non-invasive and non-destructive manner at a high resolution. In principle, three SAM approaches have reported in literature. The measurement of speed of sound in thin samples has been successfully used to study cortical and trabecular bone elastic properties in human [2]. In this method, two parallel and flat surfaces needs to be prepared for the very thin samples and additional methods are required for the measurement of density and sample thickness. In addition, this method has only been applied with frequencies up to $50 \mathrm{MHz}$ owing to the high attenuation of bone tissue. This technique found the mean elastic modulus of the cortical bone tissue of $20.6 \pm 0.2$ $\mathrm{GPa}$ in the longitudinal direction and $14.9 \pm 0.5 \mathrm{GPa}$ in the transverse direction, and that of trabecular tissue of $17.5 \pm 1.1 \mathrm{GPa}[2]$. 
In our study, the elastic modulus of cortical and trabecular bone tissue have been calculated by measuring the velocity of the LSAW. Measurement of the LSAW velocity and other mechanical properties is commonly performed using the interference method, also referred to as the $V(z)$ method. The H-SAM machine used to measure the bone mechanical properties in this study provides high image resolution due to its use of the time-gate method for wave separation. This method is based on the measurement of the interference of the longitudinal wave directly reflected from the specimen surface and the LSAW propagated along the specimen surface [17]. As the average diameter of an osteocyte is approximately $4 \mu \mathrm{m}$ [27], use of a $200-\mathrm{MHz}$ SAM machine and a spatial resolution of $7 \mu \mathrm{m}$ to evaluate the distribution of bone mechanical properties allowed for measurement of narrow trabecular bone in the osteoporosis patients examined in this study. The current study found the mean elastic modulus of cortical bone tissue to range from 14.26 to $35.28 \mathrm{GPa}$ in the longitudinal direction and from 5.07 to $13.38 \mathrm{GPa}$ in the transverse direction, and that of trabecular bone tissue to range from 14.84 to $28.17 \mathrm{GPa}$ in the longitudinal direction and from 5.24 to $10.5 \mathrm{GPa}$ in the transverse direction.

Raum et al. reported a relation which enables the estimation of anisotropic tissue elasticity of cortical bone $[8,9]$. In their method, anisotropic elastic properties were calculated from the acoustic impedance mapping of the confocal reflection amplitude. This method needs accurate sample preparation such as smooth and plane surfaces placed perpendicular to the sound beam axis and the stable measurement conditions for deriving reliable estimates of the acoustic impedance. They described that assessment of changes of local tissue anisotropy may provide new insights in studies of bone remodeling, pathologies and strength.

In cortical bone, endosteal bone resorption causes thinning of the cortex in a process known as trabecularization or cancellization with increasing age [28]. In this study, the elastic modulus of the cortical bone tissue of the lateral trochanter as low as that of trabecular bone in individuals over 70 years of age. Based on these findings, it is hypothesized that trabecularization is associated with decrease of the elastic modulus of cortical bone in the lateral trochanter.

Osteoporosis is generally believed to result when the extent of bone resorption is greater than formation, resulting in a bone remodeling imbalance and bone loss. In the stance phase of the gait cycle, a bending moment on the femoral neck generates compression on the medial side of the trochanter [29]. Several studies using finite element modeling have found that the lateral trochanter sustains tensile forces while the medial trochanter sustains high compressive forces [30, 31]. According to Wolff's law, bone formation occurs at the compressive area while bone resorption, which is more likely to occur in the proximal region of the lateral trochanter anatomically most distant 
from the medial compression area during daily walking at a normal gait, occurs at the tensile area. Based on these findings, it is hypothesized that long-term increase in bone resorption is related to the low elastic modulus of the trabecular bone in the proximal region of the lateral trochanter.

The current study had several limitations that may limit the generalizability of the results. First, this study has small number of samples in a relatively narrow age range. Second, it examined the mechanical properties of bones from individuals with no reported history of bone-related disease but for whom a detailed history, including data regarding medical and medication history and length of period of bed rest before death, was unavailable. Third, the bone specimens needed to be fixed with a $4 \%$ formalin solution for processing, embedding, and polishing. Although most studies that have investigated the effects of formalin fixation have found that it does not affect the mechanical properties of bone [32-34], several have found that it may affect bone biomechanical properties $[35,36]$. Nevertheless, fixation in a $4 \%$ formalin solution for 4 weeks has been found to have no effect on the Young's modulus, yield stress, or ultimate stress of human bone [33]. As the bone specimens in the current study were fixed in a $4 \%$ formalin solution for 4 days, it can be reasonably assumed that the embalming process had no effect on the results obtained regarding the mechanical properties of bone. Forth, the elastic modulus in the measurement of this study was obtained through the average value in any direction without regard to the anisotropy of bone tissue. However, in the purpose of this study to know the regional differentiation of elastic modulus of the trochanteric region, the assessment using even with average value of elastic modulus showed a regional difference in a characteristic manner.

In our study, the mean bone density of the trochanteric region ranged from 0.35 to $0.81 \mathrm{~g} / \mathrm{cm}^{3}$ in cortical bone tissue and from 0.38 to $0.67 \mathrm{~g} / \mathrm{cm}^{3}$ in trabecular bone tissue. The values of the bone density which were derived using equation (11) in the process of obtaining the elastic modulus were within a reasonable range as the density of bone [37, 38]. This supports the rationality of our method.

In general speaking, the mechanical properties of the bone are anisotropy. As pointed out by the authors $[39,40]$ regarding with more details of the bone mechanical anisotropic properties, the bone properties are the transversely isotropic along the gravity direction, namely the bone is isotropic, in which state equation (8) is available, in the perpendicular plane to the gravity direction and orthotropic in the parallel plane to the gravity direction, in which state equation (8) is invalid and then orthotropic elastic constitutive equations and wave equation have been applied [40]. To measure the bone anisotropic properties using the proposed SAM system, the slit has been attached to the front of the SAM acoustic lens, then the leaky surface wave velocity along the slit 
direction can be obtained. Then we can measure the anisotropic leaky surface wave velocity of the bone tissue by rotating the slit at the one point of the bone tissue [39]. It was found in the previous paper [40] that the elastic modulus of femur bone of young canine showed more anisotropy than that of elder human and the range of maximum ratio of the young canine orthotropic elastic moduli was almost 1.1-1.25. This process needs the individual measurement at the measuring points due to the rotation of the slit and so the sequential measurements like in this submitted paper are difficult at the present. However, the main purpose of our study is to clear the distribution of the elastic modulus within the same specimen of the trochanteric area of human being. The elastic modulus calculated with our method indicates the average value of the elastic modulus in any direction without considering the anisotropic properties. The elastic modulus of cortical bone in our study will be indicative of the value intermediate between the elastic modulus in the parallel plane to the gravity and that in the perpendicular plane to the gravity. Moreover, the degree of anisotropy generally decreases with age. Therefore, the regional difference of the elastic modulus in the cortical bones such as, between lateral and medial trochanters and in the trabecular bones such as, between proximal and distal areas of the lateral trochanter observed in our study will be meaningful because the measurements were made under the same condition and in the same sample. These findings will have not been neglected because of the minor anisotropy.

In trochanteric fracture, the fracture line typically passes from the proximal lateral trochanteric portion to the distal medial cortex of the medial trochanter [41]. On the other hand, magnetic resonance imaging of incomplete trochanteric fracture has indicated that the fracture line emanates from the lateral trochanter and extends into the intertrochanteric region but does not disrupt the medial cortex [42]. These findings suggest that complete trochanteric fracture begins superolaterally and propagates inferomedially in the trochanteric region. In the present study, the elastic modulus of the trabecular bone tissue of the proximal portion of the lateral trochanter was found to be low compared to that of the distal portion in all individuals aged between 70 and 89 . Based on the findings of this study and of previous studies, it is hypothesized that the proximal region of the lateral trochanter may become the weakest point with increasing age.

\section{Conclusion}

We have devised a method that can obtain continuous detailed distributions of the elastic modulus along the measurement line in a non-decalcified specimen of human bone tissue. This is the first study to investigate the local distribution of mechanical 
properties within the trochanteric region. Besides the limitation of the sample size, the findings of this study may suggest that the decrease in the elastic modulus of cortical bone in the lateral trochanter and the low value of the elastic modulus of trabecular bone in the proximal region of the lateral trochanter may be related to the increase of the rates of trochanteric fracture with age in the elderly female population. We consider that this method may contribute to studies of osteoporotic fracture, treatment of osteoporosis and bone fracture healing.

Acknowledgments The authors would like to acknowledge Seiichi Omori for the support in data acquisition.

Conflict of Interest The authors do not have any conflicts of interest to declare.

Ethical Statement All procedures followed were in accordance with the ethical standards of the responsible committee on human experimentation (institutional and national) and with the Helsinki Declaration of 1975, as revised in 2008 (5). Informed consent was obtained from all patients for being included in the study. 


\section{References}

1. Rho JY, Tsui TY, Pharr GM. Elastic properties of human cortical and trabecular lamellar bone measured by nanoindentation. Biomaterials. 1997;18:1325-30.

2. Turner $\mathrm{CH}$, Rho J, Takano Y, et al. The elastic properties of trabecular and cortical bone tissues are similar: results from two microscopic measurement techniques. J Biomech. 1999;32:437-41.

3. Zysset PK, Guo XE, Hoffler CE, et al. Elastic modulus and hardness of cortical and trabecular bone lamellae measured by nanoindentation in the human femur. J Biomech. 1999;32:1005-12.

4. Hou FJ, Lang SM, Hoshaw SJ, et al. Human vertebral body apparent and hard tissue stiffness. J Biomech. 1998;31:1009-15.

5. Ladd AJ, Kinney JH, Haupt DL, et al. Finite-element modeling of trabecular bone: comparison with mechanical testing and determination of tissue modulus. J Orthop Res. 1998;16:622-8.

6. Niebur GL, Feldstein MJ, Yuen JC, et al. High-resolution finite element models with tissue strength asymmetry accurately predict failure of trabecular bone. $\mathrm{J}$ Biomech. 2000;33:1575-83.

7. Bayraktar HH, Morgan EF, Niebur GL, et al. Comparison of the elastic and yield properties of human femoral trabecular and cortical bone tissue. J Biomech. 2004;37:27-35.

8. Raum K, Cleveland RO, Peyrin F, et al. Derivation of elastic stiffness from site-matched mineral density and acoustic impedance maps. Phys Med Biol. 2006;51:747-58.

9. Raum K. Microelastic imaging of bone. IEEE Trans Ultrason Ferroelectr Freq Control. 2008;55:1417-31.

10. Granke M, Grimal Q, Saïed A, et al. Change in porosity is the major determinant of the variation of cortical bone elasticity at the millimeter scale in aged women. Bone 2011;49:1020-6.

11. Malo MK, Rohrbach D, Isaksson H, et al. Longitudinal elastic properties and porosity of cortical bone tissue vary with age in human proximal femur. Bone. 2013;53:451-8.

12. Giversen IM. Time trends of mortality after first hip fractures. Osteoporos Int. 2007; 18:721-32.

13. Braithwaite RS, Col NF, Wong JB. Estimating hip fracture morbidity, mortality and costs. J Am Geriatr Soc. 2003;51:364-70.

14. Kannus P, Parkkari J, Sievänen H, et al. Epidemiology of hip fractures. Bone 
1996;18 (1 Suppl):57S-63S.

15. Hagino H, Furukawa K, Fujiwara S, et al. Recent trends in the incidence and lifetime risk of hip fracture in Tottori, Japan. Osteoporos Int. 2009;20:543-8.

16. Parmon W, Bertoni HL. Ray Interpretation of the Material Signature in the Acoustic Microscope. Electronics Letters. 1979;15:684-6.

17. Ishikawa I, Katakura K, Ogura Y. Full-circular surface acoustic wave excitation for high resolution acoustic microscopy using spherical lens and time gate technology. IEEE Trans Ultrason Ferroelectr Freq Control. 1999;46:41-6.

18. Kobayashi M. Japan Patent Application Number: 2000-028592, 2000.

19. Nakatsuchi Y, Tateiwa H, Kobayashi M, et al. Detection of the microcracks in the human trochanteric region using a scanning acoustic microscope. Nihon Rinshou Biomechanics Gakkaishi (Japanese Journal of Clinical Biomechanics). 2001;22:269-74 (in Japanese).

20. Fung YC. Foundations of Solid Mechanics. Prentice-Hall, Englewood Cliffs, NJ; 1965. p. 178-81.

21. Shibano J, Kobayashi M, Omori S. Quick Measurement Method of Microscopic Distribution of Mechanical Property on Solid Surface using Scanning Acoustic Microscope. Chouonpa techno (Ultrasonic Technology). 2006;18:108-11 (in Japanese).

22. Fan Z, Rho JY. Effects of viscoelasticity and time-dependent plasticity on nanoindentation measurements of human cortical bone. J Biomed Mater Res A. 2003;67:208-14.

23. Van Rietbergen B, Odgaard A, Kabel J, et al. Relationships between bone morphology and bone elastic properties can be accurately quantified using high-resolution computer reconstructions. J Orthop Res. 1998;16:23-8.

24. Keaveny TM, Pinilla TP, Crawford RP, et al. Systematic and random errors in compression testing of trabecular bone. J Orthop Res. 1997;15:101-10.

25. Jacobs CR, Davis BR, Rieger CJ, et al. The impact of boundary conditions and mesh size on the accuracy of cancellous bone tissue modulus determination using large-scale finite-element modeling. North American Congress on Biomechanics. J Biomech. 1999;32:1159-64.

26. Lemons RA, Quate CF. Acoustic microscopy: biomedical applications. Science. 1975;188:905-11.

27. Wang X, Shen X, Li X, et al. Age-related changes in the collagen network and toughness of bone. Bone. 2002;31:1-7.

28. Bell KL, Loveridge N, Power J, et al. Regional differences in cortical porosity in the fractured femoral neck. Bone. 1999;24:57-64. 
29. Duda GN, Schneider E, Chao EY. Internal forces and moments in the femur during walking. J Biomech. 1997;30:933-41.

30. Van Rietbergen B, Huiskes R, Eckstein F, et al. Trabecular bone tissue strains in the healthy and osteoporotic human femur. J Bone Miner Res. 2003;18:1781-8.

31. Rudman KE, Aspden RM, Meakin JR. Compression or tension? The stress distribution in the proximal femur. Biomed Eng Online. 2006;20:12.

32. Pöpperl G, Lochmüller E, Becker H, et al. Determination of calcaneal ultrasound properties ex situ: reproducibility, effects of storage, formalin fixation, maceration, and changes in anatomic measurement site. Calcif Tissue Int. 1999;65:192-7.

33. Ohman C, Dall'Ara E, Baleani M, et al. The effects of embalming using a 4\% formalin solution on the compressive mechanical properties of human cortical bone. Clin Biomech (Bristol, Avon). 2008;23:1294-8.

34. Nazarian A, Hermannsson BJ, Muller J, et al. Effects of tissue preservation on murine bone mechanical properties. J Biomech. 2009;42:82-6.

35. Currey JD, Brear K, Zioupos P, et al. Effect of formaldehyde fixation on some mechanical properties of bovine bone. Biomaterials. 1995;16:1267-71.

36. Burkhart KJ, Nowak TE, Blum J, et al. Influence of formalin fixation on the biomechanical properties of human diaphyseal bone. Biomed Tech. 2010;55:361-5.

37. Akhter MP, Lappe JM, Davies KM, et al. Transmenopausal changes in the trabecular bone structure. Bone. 2007;41:111-6.

38. Mödder UI, Achenbach SJ, Amin S, et al. Relation of serum serotonin levels to bone density and structural parameters in women. J Bone Miner Res. 2010;25:415-22.

39. Nakatsuchi Y, Kobayashi M, Nomura A, et al. Acoustic anisotropy of normal and healing cortical bone. Nihon Rinshou Biomechanics Gakkaishi (Japanese Journal of Clinical Biomechanics). 1999;20:213-7 (in Japanese).

40. Kobayashi M, Shibano J, Nakatsuchi Y, et al. Anisotropic mechanical properties of femur bone measured by scanning acoustic microscope. Transactions of the Japan Society of Mechanical Engineers. 2006;72:1072-9 (in Japanese).

41. Jensen JS. Classification of trochanteric fractures. Acta Orthop Scand. 1980;51:803-10.

42. Schultz E, Miller TT, Boruchov SD, et al. Incomplete intertrochanteric fractures: imaging features and clinical management. Radiology. 1999;211:237-40. 


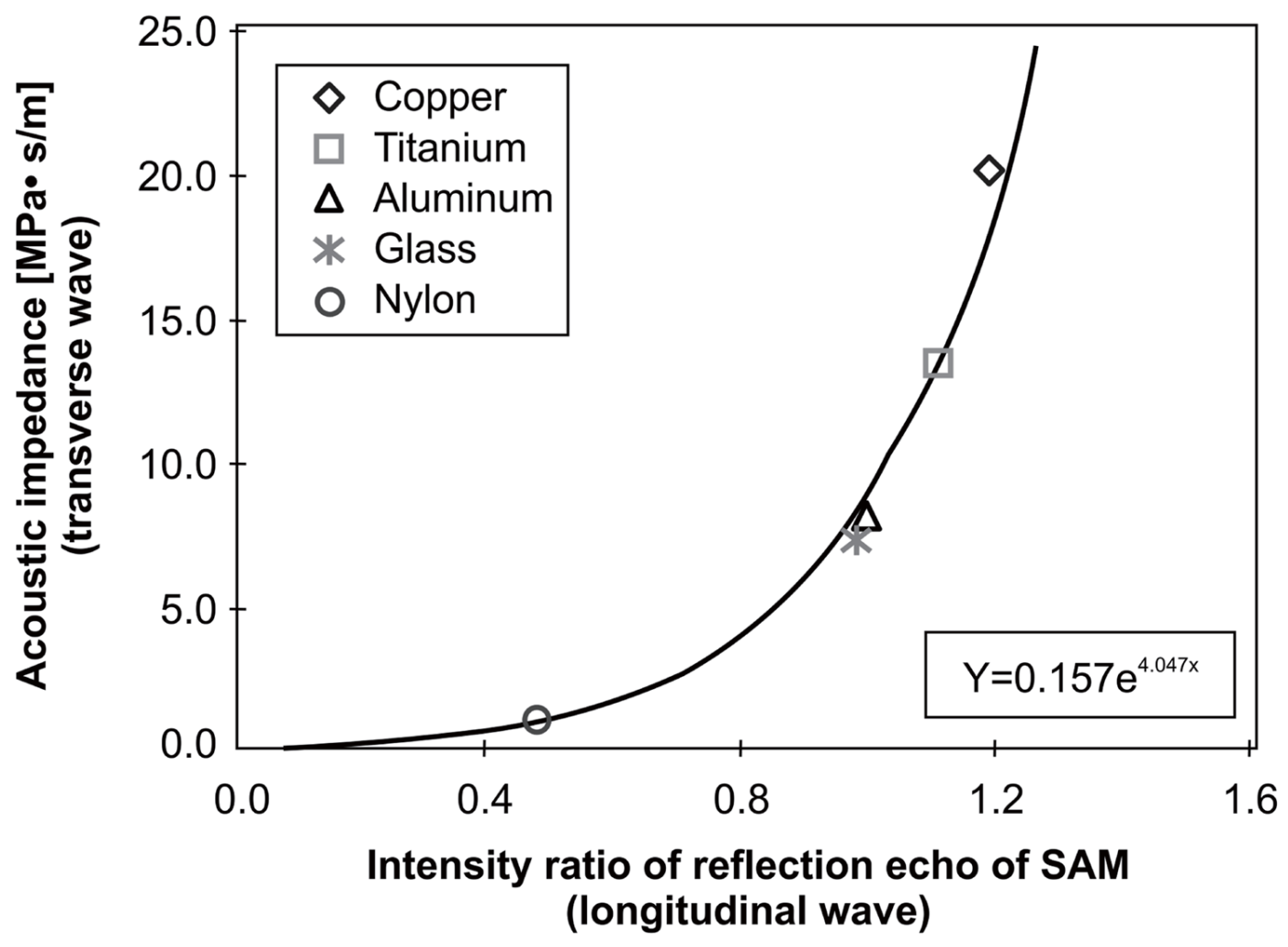

Fig. 1 The approximate curve of the acoustic impedance in transverse wave versus reflection intensity ratio in longitudinal wave. The relationship between acoustic impedance in transverse wave $Z_{T}$ and reflection intensity ratio in longitudinal wave $X$ was plotted based on the known values of several metals and non-metals. From this relationship, the approximate curve and its equation, $\mathrm{Y}=0.157 \mathrm{e}^{4.047 \mathrm{X}}$ were obtained. 


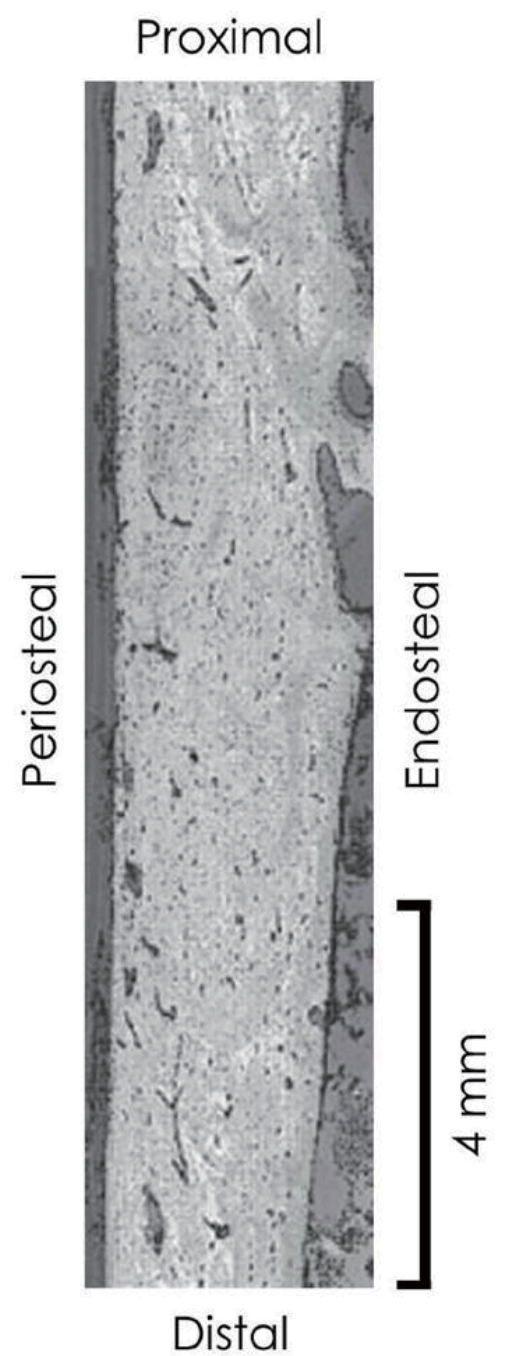

Fig. 2 C-mode image of the coronal section of the canine femoral cortical bone. 


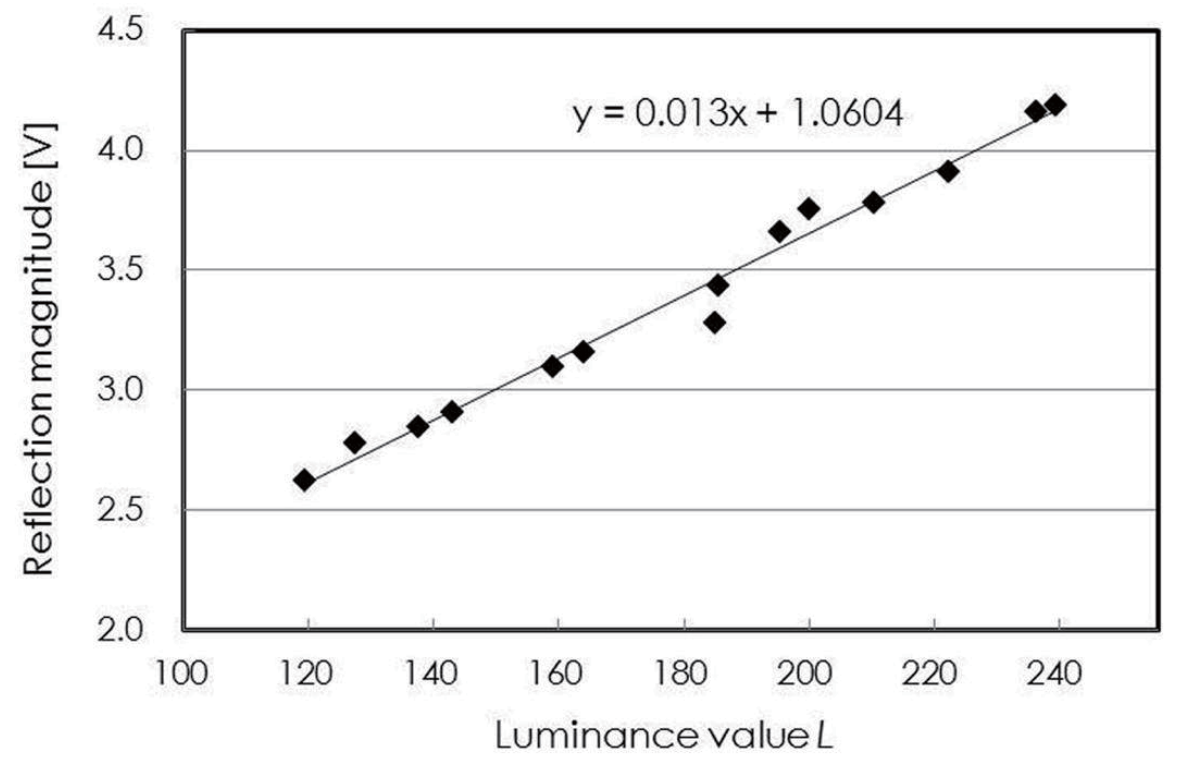

Fig. 3 The relation between the luminance value of C-mode image and ultrasonic reflection magnitude of the bone specimen. 


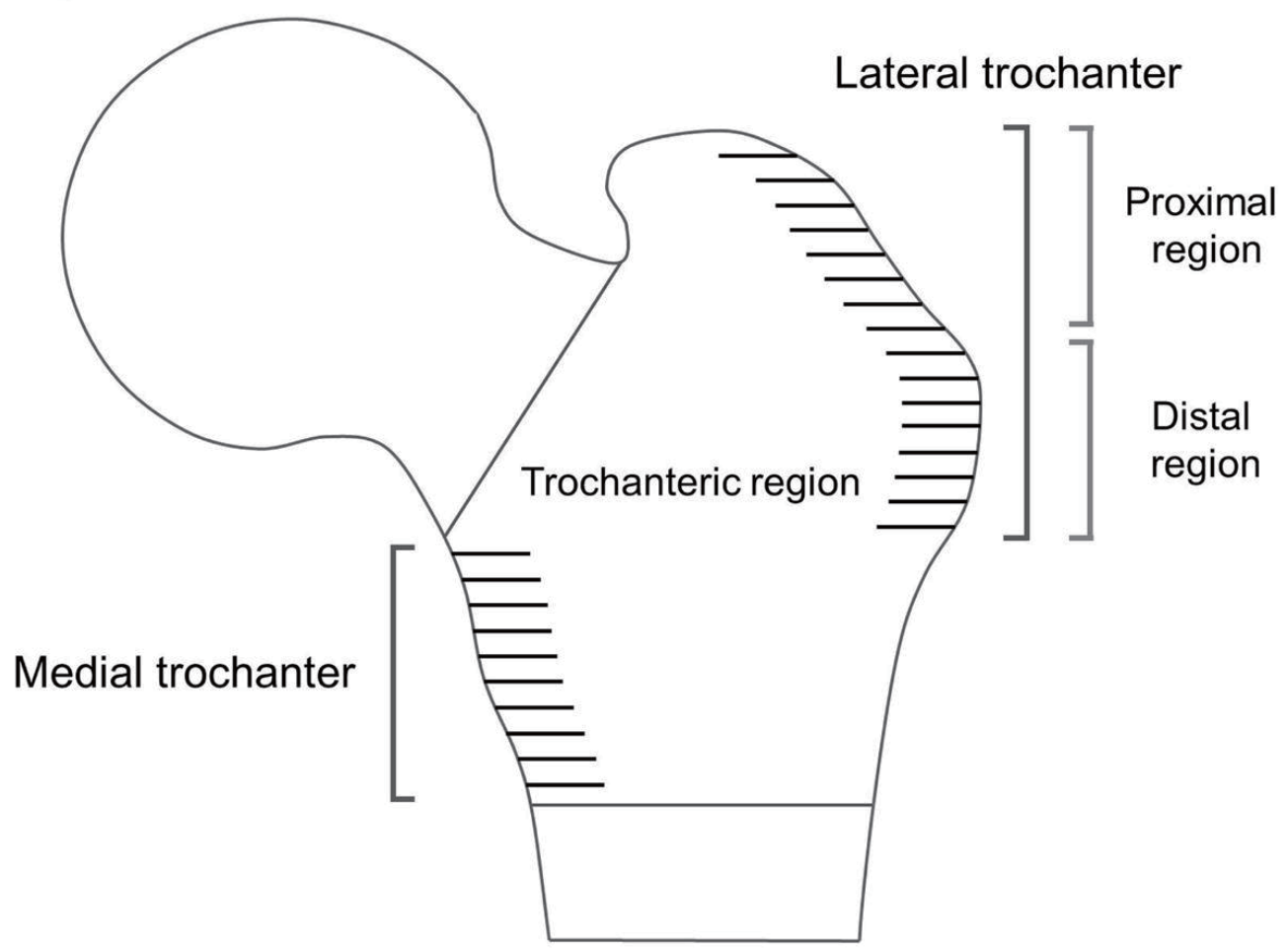

Fig. 4 Measurement of the elastic modulus. In the lateral trochanter, cortical and trabecular bones were scanned perpendicular to the axis of femoral shaft from the tip of the greater trochanter to the level of the upper rim of the lesser trochanter, with 16 lines scanned with each $4 \mathrm{~mm}$ scan length at regular intervals. In the medial trochanter, 10 lines were scanned at the cortical and trabecular bones perpendicular to the axis of the femoral shaft from the upper rim to the lower rim of the lesser trochanter at regular intervals. 

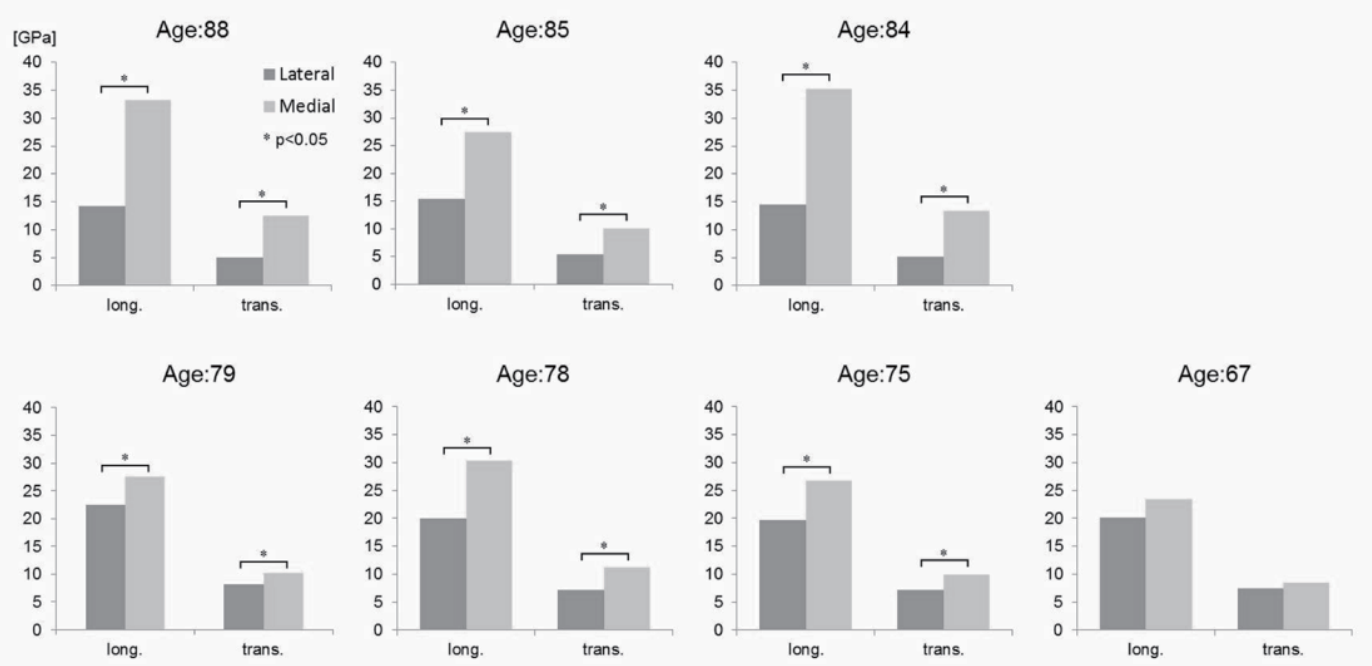

Fig. 5 The elastic modulus of the cortical bone tissue in the trochanteric region. The longitudinal and transverse elastic moduli of the cortical bone tissue of the lateral trochanter of all specimens from individuals over age 70 were found to be significantly lower than that of the medial trochanter. long.: longitudinal, trans.: transverse, Lateral: lateral trochanter, Medial: medial trochanter 

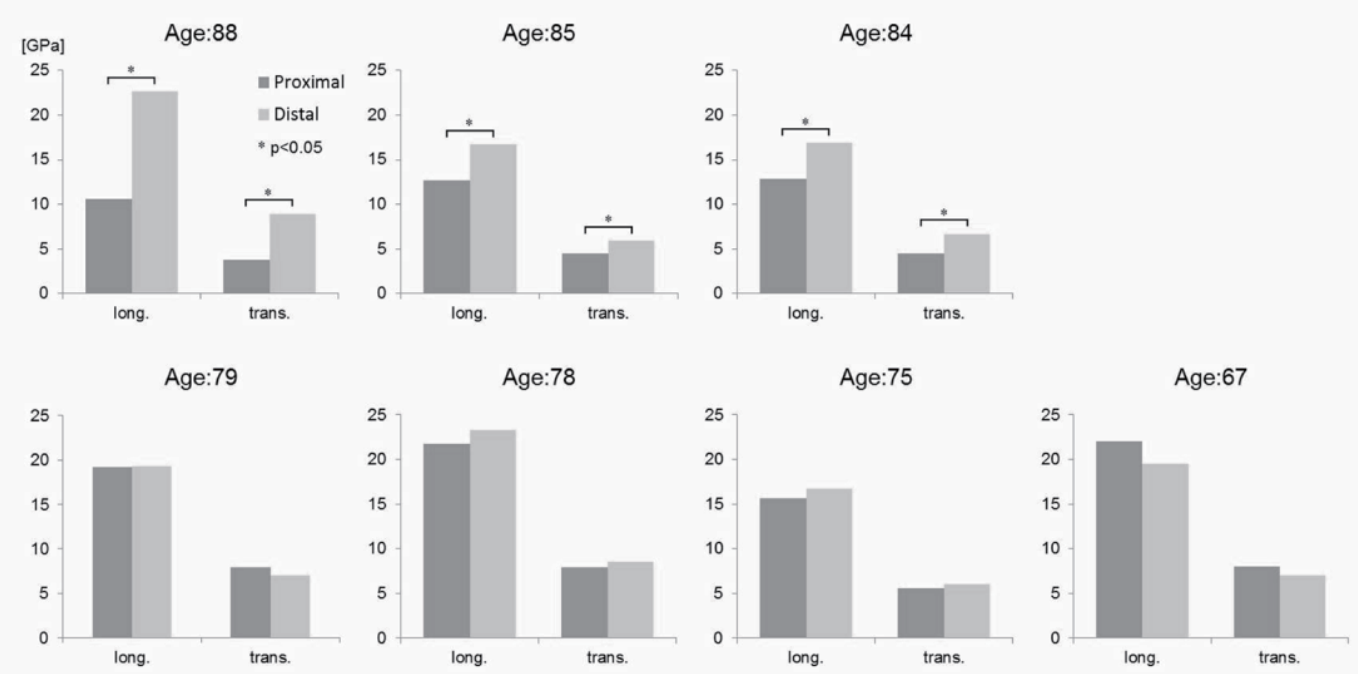

Fig. 6 The elastic modulus of the trabecular bone tissue in the lateral trochanter. The longitudinal and transverse elastic moduli of the trabecular bone tissue of the proximal region of all individuals aged between 80 and 89 were found to be significantly lower than that of the distal region. long.: longitudinal, trans.: transverse, Proximal: proximal region of the lateral trochanter, Distal: distal region of the lateral trochanter 


\section{Table 1}

Measurement area of trochanteric regions

\begin{tabular}{ccccc}
\hline \multicolumn{2}{c}{ Specimen } & & \multicolumn{2}{c}{ Longitudinal length of measurement area } \\
\cline { 1 - 2 } \cline { 4 - 4 } Age & sex & & Lateral trochanter $(\mathrm{mm})$ & Medial trochanter $(\mathrm{mm})$ \\
\hline 88 & female & 46 & 27 \\
85 & female & 48 & 29 \\
84 & female & 47 & 29 \\
79 & female & 46 & 26 \\
78 & female & 44 & 30 \\
75 & female & 48 & 27 \\
67 & female & 46 & 29 \\
\hline
\end{tabular}

Lateral trochanter was measured from the tip of the greater trochanter to the level of the upper rim of the lesser trochanter.

Medial trochanter was measured from the upper rim to the lower rim of the lesser trochanter. 


\section{Table 2}

Results of elastic modulus and bone density in the trochanteric region.

\begin{tabular}{|c|c|c|c|c|c|c|c|}
\hline \multicolumn{2}{|c|}{ Donor } & \multicolumn{3}{|c|}{ Cortical bone tissue } & \multicolumn{3}{|c|}{ Trabecular bone tissue } \\
\hline Age & Region & $\begin{array}{l}\text { Elastic modulus } \\
\text { (long., in GPa) }\end{array}$ & $\begin{array}{l}\text { Elastic modulus } \\
\text { (trans., in GPa) }\end{array}$ & Density $\left(\mathrm{g} / \mathrm{cm}^{3}\right)$ & $\begin{array}{l}\text { Elastic modulus } \\
\text { (long., in GPa) }\end{array}$ & $\begin{array}{l}\text { Elastic modulus } \\
\text { (trans., in GPa) }\end{array}$ & Density $\left(\mathrm{g} / \mathrm{cm}^{3}\right)$ \\
\hline \multirow[t]{2}{*}{88} & Lateral & $14.26(6.26)^{*}$ & $5.07(2.35)^{*}$ & $0.35(0.15)^{*}$ & $17.02(10.94)$ & $6.17(4.15)$ & $0.39(0.22)$ \\
\hline & Medial & $33.20(6.61)$ & $12.47(2.74)$ & $0.76(0.19)$ & $23.72(7.03)$ & $8.76(2.64)$ & $0.58(0.13)$ \\
\hline \multirow[t]{2}{*}{85} & Lateral & $15.47(3.62)^{*}$ & $5.46(1.32)^{*}$ & $0.36(0.10)^{*}$ & $14.84(2.23)^{*}$ & $5.24(0.84)^{*}$ & $0.38(0.09)^{*}$ \\
\hline & Medial & $27.35(7.51)$ & $10.06(2.97)$ & $0.60(0.17)$ & $24.92(5.04)$ & $9.10(2.01)$ & $0.60(0.16)$ \\
\hline \multirow[t]{2}{*}{84} & Lateral & $14.56(6.10)^{*}$ & $5.21(2.26)^{*}$ & $0.42(0.17)^{*}$ & $14.88(4.27)^{*}$ & $5.58(1.45)^{*}$ & $0.40(0.12)$ \\
\hline & Medial & $35.28(8.21)$ & $13.38(3.31)$ & $0.81(0.18)$ & $24.66(9.13)$ & $9.19(2.11)$ & $0.58(0.30)$ \\
\hline \multirow[t]{2}{*}{79} & Lateral & $22.53(5.04)^{*}$ & $8.23(1.98)^{*}$ & $0.59(0.16)$ & $19.29(4.52)^{*}$ & $7.45(2.54)^{*}$ & $0.53(0.13)$ \\
\hline & Medial & $27.59(6.01)$ & $10.18(2.44)$ & $0.66(0.14)$ & $25.38(4.21)$ & $9.31(1.67)$ & $0.67(0.07)$ \\
\hline \multirow[t]{2}{*}{78} & Lateral & $19.96(6.03)^{*}$ & $7.18(2.31)^{*}$ & $0.49(0.16)^{*}$ & $22.55(4.91)$ & $8.18(1.90)$ & $0.54(0.10)$ \\
\hline & Medial & $30.30(6.44)$ & $11.27(2.66)$ & $0.73(0.18)$ & $28.17(9.15)$ & $10.50(5.23)$ & $0.64(0.30)$ \\
\hline \multirow[t]{2}{*}{75} & Lateral & $19.68(4.41)^{*}$ & $7.13(1.69)^{*}$ & $0.50(0.12)^{*}$ & $16.16(2.00)$ & $5.79(0.75)$ & $0.43(0.08)$ \\
\hline & Medial & $26.79(7.74)$ & $9.96(3.03)$ & $0.66(0.18)$ & $18.27(9.94)$ & $6.67(3.8)$ & $0.45(0.22)$ \\
\hline \multirow[t]{2}{*}{67} & Lateral & $20.10(4.63)$ & $7.47(1.91)$ & $0.54(0.14)$ & $20.76(4.37)$ & $7.51(1.67)$ & $0.55(0.08)$ \\
\hline & Medial & $23.40(4.81)$ & $8.52(1.89)$ & $0.55(0.11)$ & $23.10(4.37)$ & $8.41(1.72)$ & $0.58(0.10)$ \\
\hline
\end{tabular}

Values are means and (standard deviation).

long., longitudinal; trans., transverse; Lateral; lateral trochanter; Medial, medial trochanter;

${ }^{*} \mathrm{p}<0.05$ compared with corresponding Medial. 


\section{Table 3}

Elastic modulus and bone density of trabecular bone tissue at the lateral trochanter.

\begin{tabular}{|c|c|c|c|c|}
\hline \multicolumn{2}{|c|}{ Donor } & \multicolumn{3}{|c|}{ Trabecular bone tissue } \\
\hline Age & Region & $\begin{array}{l}\text { Elastic modulus } \\
\text { (long., in GPa) }\end{array}$ & $\begin{array}{l}\text { Elastic modulus } \\
\text { (trans., in GPa) }\end{array}$ & Density $\left(\mathrm{g} / \mathrm{cm}^{3}\right)$ \\
\hline \multirow[t]{2}{*}{88} & Proximal & $10.62(7.49) *$ & $3.76(2.56)^{*}$ & $0.23(0.09)^{*}$ \\
\hline & Distal & $22.61(8.72)$ & $8.92(3.99)$ & $0.55(0.19)$ \\
\hline \multirow[t]{2}{*}{85} & Proximal & $12.67(1.63)^{*}$ & $4.45(0.59)^{*}$ & $0.34(0.05)^{*}$ \\
\hline & Distal & $16.73(2.34)$ & $5.92(0.89)$ & $0.42(0.11)$ \\
\hline \multirow[t]{2}{*}{84} & Proximal & $12.85(2.42)^{*}$ & $4.51(0.97)^{*}$ & $0.32(0.07)^{*}$ \\
\hline & Distal & $16.90(4.89)$ & $6.66(0.98)$ & $0.48(0.12)$ \\
\hline \multirow[t]{2}{*}{79} & Proximal & $19.25(4.91)$ & $7.90(3.21)$ & $0.53(0.13)$ \\
\hline & Distal & $19.32(4.49)$ & $6.98(1.74)$ & $0.53(0.13)$ \\
\hline \multirow[t]{2}{*}{78} & Proximal & $21.79(3.09)$ & $7.88(1.21)$ & $0.54(0.09)$ \\
\hline & Distal & $23.31(6.39)$ & $8.49(2.46)$ & $0.54(0.03)$ \\
\hline \multirow[t]{2}{*}{75} & Proximal & $15.64(2.35)$ & $5.60(0.88)$ & $0.39(0.10)$ \\
\hline & Distal & $16.75(1.48)$ & $6.02(0.55)$ & $0.46(0.13)$ \\
\hline \multirow[t]{2}{*}{67} & Proximal & $21.99(3.39)$ & $7.97(1.29)$ & $0.59(0.05)$ \\
\hline & Distal & $19.52(5.10)$ & $7.04(1.96)$ & $0.51(0.14)$ \\
\hline
\end{tabular}

Values are means and (standard deviation).

Proximal: proximal region of lateral trochanter, Distal: distal region of lateral trochanter, ${ }^{*}$ p $<0.05$ compared with corresponding Distal. 